\title{
Evaluation of trigger tool method for adverse drug reaction reporting by nursing staff at a tertiary care teaching hospital
}

\author{
Urmila J. Menat ${ }^{1}$, Chetna K. Desai' ${ }^{2}$, Megha K. Shah ${ }^{2 *}$, Asha N. Shah ${ }^{3}$
}

${ }^{1}$ Drug Safety Physician, APCER Life Science, Ahmedabad, Gujarat, India

${ }^{2}$ Department of Pharmacology, BJ Medical College, Ahmedabad, Gujarat, India ${ }^{3}$ Department of Medicine, GCS Medical College, Ahmedabad, Gujarat, India

Received: 28 March 2019

Revised: 19 April 2019

Accepted: 03 May 2019

*Correspondence to:

Dr. Megha K. Shah,

Email: mkshah_80@yahoo.com

Copyright: (c) the author(s), publisher and licensee Medip Academy. This is an openaccess article distributed under the terms of the Creative Commons Attribution NonCommercial License, which permits unrestricted noncommercial use, distribution, and reproduction in any medium, provided the original work is properly cited.

\begin{abstract}
Background: To sensitize nurses about Trigger Tool Method (TTM) and to evaluate the impact of TTM on adverse drug event (ADE) reporting by nurses at a tertiary care teaching hospital in India.

Methods: This was prospective, interventional, single center study conducted among nursing health professionals of Civil Hospital Ahmedabad (CHA) posted in Medicine Department. They were sensitized about ADE reporting, pharmacovigilance, methods of ADRs reporting and details about TTM. Also, a list of 17 triggers was prepared by the investigator and given to nurses. They were educated to report ADEs using TTM. At the initiation and end of study, questionnaires were given to evaluate knowledge, attitude and practice of ADR reporting among participant nurses. All triggers and ADEs reported were analyzed in terms of association between them, effectiveness of trigger in detecting an ADR and in terms of Positive Predictive Value (PPV). Reported ADRs were also assessed for causality, severity and preventability.

Results: A total 758 patients were admitted during the study period in the respective medicine department. List of 17 triggers consists of 9 drug triggers (DT), 1 laboratory trigger (LT) and 7 patient triggers (PT). Of these 17 triggers, 14 triggers were identified by nurses. These 14 triggers were noticed 130 times. These included DT (100 times), LT (0 times) and PT (30 times). Of the various triggers observed, 7 DT and 4 PT were related to ADRs. Hence, 11 triggers $(64.70 \%)$ were positive (related to ADRs), out of 17 total triggers under evaluation. 21 ADRs were observed using TTM by nurses.
\end{abstract}

Conclusions: The TTM helps to detect and report ADRs by nurses. Educational interventions about TTM help in better detection and reporting of ADRs.

Keywords: Adverse drug event, Adverse drug reaction reporting, Nursing health professionals, Pharmacovigilance, PvPI, Trigger tool method

\section{INTRODUCTION}

Adverse drug reactions (ADRs) defined as "a response to drug that is noxious, unintended and occur in doses used in human beings for prophylaxis, diagnosis and therapy of disease or for modification of physiological function". ${ }^{1}$ According to a study conducted in USA, about 2.9-5.6\% of all hospitalizations were due to ADRs and as many as $35 \%$ of hospitalized patients experienced an ADR during their stay. ${ }^{2}$ A study conducted in south India observed that while $0.7 \%$ of the hospital admissions were due to ADRs,
$3.7 \%$ of the hospitalized patients experienced an ADR and $1.8 \%$ had fatal ADRs during hospitalization. ${ }^{3}$

There are several methods to monitor ADRs like voluntary reporting, record review, triggers, direct observation, interviews/surveys, targeted reporting, cohort event monitoring, HER mining (electronic health record mining). ${ }^{4}$ Voluntary reporting of ADRs is most commonly used method for reporting of ADRs. However, voluntary reporting has some disadvantages like under reporting, reporting bias, difficult to detect delayed ADRs and 
capture only suspected ADRs. So, other methods needed to improve reporting of ADRs. ${ }^{4}$ One of them is trigger tool method (TTM).

A trigger is defined as an "occurrence, prompt or flag found on review of the medical record that 'triggers' further investigation to determine the presence or absence of an adverse event" a trigger may include Laboratory Trigger (LT), Drug Trigger (LT), and Patient Trigger (PT). An Adverse drug event (ADE) trigger tool makes chart review more efficient by identifying suspected $\mathrm{AE}$ via laboratory values, text phrases or automated 'values' available in medical records, which is more time effective than complete chart review and more sensitive than voluntary reporting. ${ }^{4-6}$ In the 1990 , the Institute for Healthcare Improvement (IHI) developed the IHI Global trigger tool to quantify $\mathrm{AE} .^{7}$

All health care professionals including physicians, nurses and pharmacists can play an important role in detecting and reporting ADRs. Nurses are in a unique position of detecting and reporting ADRs because they are in close contact with the patients and monitoring the hospitalized patients constantly. Considering these facts, the present study had been designed to educate them about trigger tool method of ADR monitoring and reporting among nursing staff and to assess the impact of educational interventions on ADR reporting by these health professionals.

\section{METHODS}

This was prospective, interventional, single center study conducted in nursing health professionals, currently employed at Civil Hospital Ahmedabad (CHA), Gujarat, India on a permanent basis, posted in Department of Medicine. The study was conducted after permissions from the Institutional Ethics Committee, the medical superintendent and the nursing superintendent.

\section{Inclusion criteria}

- Nurses, who consent (written) to participate in the study, were included in the study.

- Nursing health professionals of two Medicine wards during the study period were included.

\section{Exclusion criteria}

- Nurses not willing to participate in the study.

- Nurses appointed as adhoc basis were excluded.

\section{Study period}

The study was conducted in two medicine wards of CHA from December 2016 to May 2017, for 5 months.

Knowledge, attitude and practice questionnaire was given to each nurse at initiation of the study. It was pretested and validated questionnaire prepared by investigator. In $1^{\text {st }}$ week they were sensitized about pharmacovigilance, methods of ADE reporting and details about trigger tool method (personal briefing, lectures). Also, a list of triggers was prepared from IHI Global Trigger Tool list (7) and list adopted by Abideen P (Indian study) and given to nurses. ${ }^{6}$ By next 2 weeks, they were advised to report ADEs using trigger tool method under guidance of investigator. In following month, they were advised to report ADEs using trigger tool method. The investigator had evaluated all reported ADEs. Reminders to report were sent 1 SMS/week. After end of study KAP questionnaires were again given to evaluate knowledge, attitude and practice of ADR reporting.

Data was analyzed in Microsoft Excel sheet. All triggers and AEs reported were analyzed in terms of association between them, effectiveness of trigger in detecting an ADR. The Positive Predictive Value (PPV) was calculated for the sets of triggers and for each trigger. PPV was defined as number of patients for whom a trigger was indicating an ADE found, divided by the number of patients for whom a trigger was indicating an ADE found plus the number of patients for whom a trigger did not indicate an ADE. ${ }^{8,9}$

PPV $=$ Number of medical records in which the trigger indicated an ADE $\times 100$ / Number of medical records with triggers.

For ADRs causality assessment was done by investigator using WHO-UMC scale and Naranjo's algorithm. ${ }^{10,11}$ Severity was assessed using modified Hartwig and Seigel while preventability was assessed using modified Schumock and Thornton scale. ${ }^{12,13}$

\section{RESULTS}

A total 758 patients were admitted during the study period in the respective medicine unit. Mean age was $41.72 \pm 18.08$ years, and mean length of hospital stay was $4.57 \pm 3.34$ days.

A list of 17 triggers was given to 30 nurses for identification of ADEs. List of 17 triggers consists of 9 DT, 1 LT and 7 PT. Of these 17 triggers, 14 triggers were identified by nurses in the study population and 3 triggers were not observed. These 14 triggers were noticed 130 times, with an average 12.53 triggers observed per patient. These included DT (100 times), LT (0 times) and PT (30 times) (Table 1). Triggers were identified for a minimum once and maximum 3 times in 95 patients. Of the various triggers observed, 7 drug triggers and 4 patient triggers were related to ADRs. Hence 11 triggers (64.70\%) were positive (related to ADRs), out of total 17 triggers under evaluation (Figure 1).

Of the 758 patient cases, triggers were observed in 95 patients (12.53\%). Among 95 patient's cases with triggers, $79(77.89 \%)$ patients did not suffer from an ADR, while 21 $(22.11 \%)$ suffered one or more ADRs. 
Table 1: Trigger tool list and positive predictive value (PPV) of triggers.

\begin{tabular}{|lllll|}
\hline Trigger & $\begin{array}{l}\text { Trigger } \\
\text { observed }\end{array}$ & $\begin{array}{l}\text { Negative triggers } \\
\text { (not related to ADRs) }\end{array}$ & $\begin{array}{l}\text { Positive triggers } \\
\text { (related to ADRs) }\end{array}$ & $\begin{array}{l}\text { Positive predictive } \\
\text { value (PPV \%) }\end{array}$ \\
\hline DT1- New drug administration & 18 & 17 & 1 & $5.55 \%$ \\
\hline DT2- Sudden stoppage of drug & 7 & 4 & 3 & $32.85 \%$ \\
\hline DT3- Antihistaminics & 6 & 4 & 2 & $33.33 \%$ \\
\hline DT4- Antiemetics & 26 & 24 & 2 & $21.4 \%$ \\
\hline DT5- Antidiarrhoeals & 14 & 11 & 3 & $17.39 \%$ \\
\hline DT6- Antacids & 23 & 19 & 4 & - \\
\hline DT7- Laxatives & 0 & 0 & 0 & 0 \\
\hline DT8- Thrombophob gel & 5 & 1 & 4 & - \\
\hline DT9- IV fluid & 1 & 1 & 0 & $28.57 \%$ \\
\hline LT1- Increased serum creatinine & 0 & 0 & 0 & $50 \%$ \\
\hline PT1- Rash & 7 & 5 & 2 & 0 \\
\hline PT2- Pruritus & 2 & 1 & 1 & 0 \\
\hline PT3- Lethargy & 3 & 3 & 0 & - \\
\hline PT4- Death & 9 & 9 & 0 & $100 \%$ \\
\hline $\begin{array}{l}\text { PT5- Transfer/reference to other } \\
\text { department }\end{array}$ & 0 & 0 & 1 & $50 \%$ \\
\hline PT6- Weight gain & 1 & 0 & 4 & \\
\hline $\begin{array}{l}\text { PT7- Other complain that are not related } \\
\text { to the disease }\end{array}$ & 8 & 4 & & 0 \\
\hline
\end{tabular}

TT- Trigger Tool, DT-Drug Trigger, LT- Laboratory Trigger, PT- Patient trigger, PPV- Positive Predictive Value.

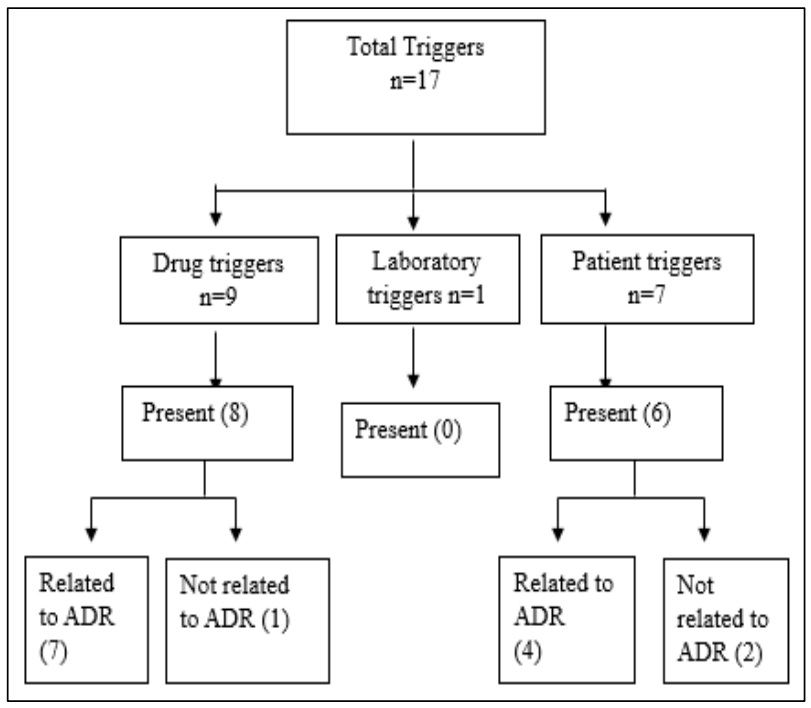

Figure 1: Number of triggers used, identified and associated with ADRs (n=number of triggers).

The PPV was calculated for each trigger and trigger group. Positive triggers observed were sudden stoppage of drug, new drug administration, antihistamines, antiemetics, thrombophob gel, blood/blood product transfusion, rash, pruritus, weight gain etc. The PPV for individual trigger tool ranged from $0 \%$ to $100 \%$. The highest PPV was for weight gain $(100 \%)$ followed by thrombophob gel $(80 \%)$, other complaints not related to disease $(50 \%)$ and sudden stoppage of drug (42.85\%) (Table 1). The overall PPV for trigger tool was $20.76 \%$.
A total 22 ADRs were observed by nurses in 21 inpatients during study period. One or more triggers were observed in these patients. The commonly detected ADRs were gastritis (5), thrombophlebitis (4), diarrhea (2) and vomiting (2). Chills, cough, headache, joint pain, metallic taste, pruritus and weight gain were also observed. Most common systems affected by ADRs were the gastrointestinal tract (10 ADEs) followed by injection site disorder (4 ADEs) and skin and appendages disorder (3 ADEs) (Figure 2).

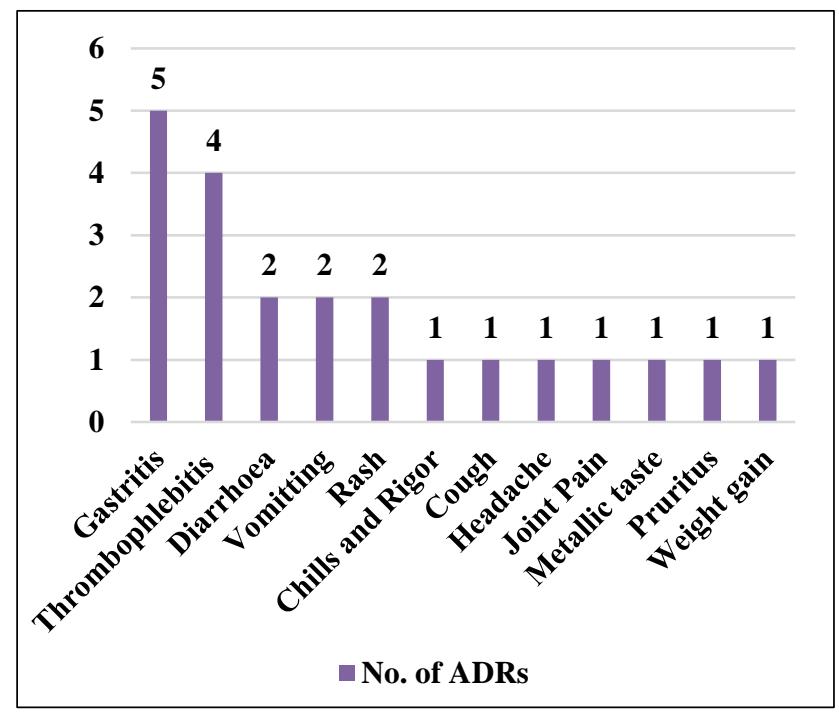

Figure 2: Detected adverse drug reactions $(n=22)$ with the help of trigger tool method. 
Blood products and non-steroidal anti-inflammatory drugs were the most common causal drug groups for ADRs. Other drug groups causing ADRs were, anti-tubercular drugs, antibiotics followed by antiretrovirals. One ADR was also reported with each antiepileptics, benzodiazepines, angiotensin converting enzyme inhibitors and hematinics group of drugs (Figure 3). The causality grade of observed ADRs was probable (18) and possible (4).

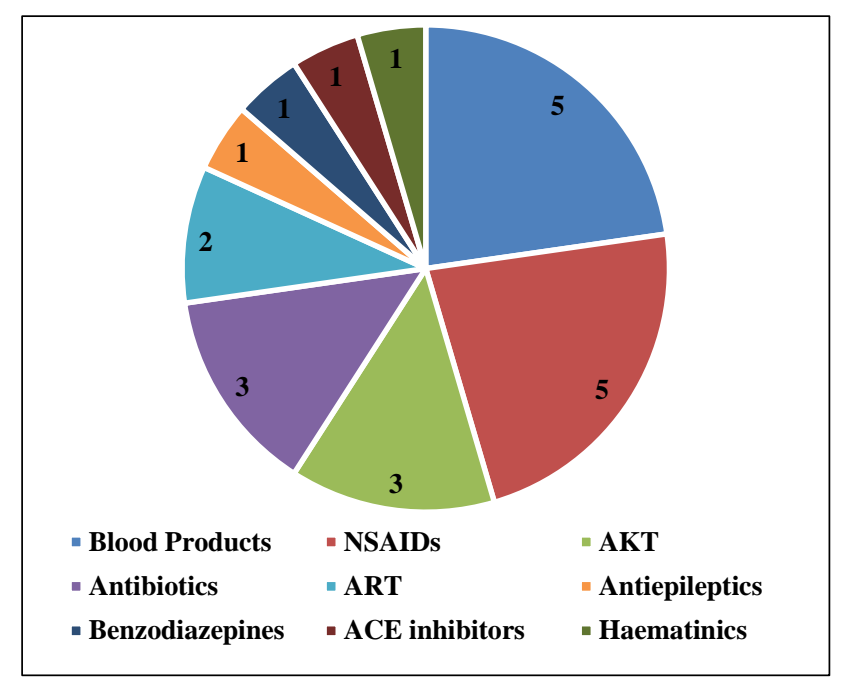

Figure 3: Common drug groups causing adverse drug reactions $(n=22)$.

A questionnaire-based test was taken before the study (pretest) and after the study (post-test) to see the awareness, attitude and practice about ADRs reporting among participant nurses.

All the 30 participant nurses $(100 \%)$ responded in pre and post-test questionnaires. At pre-test, $56.66 \%$ of nurses were unaware about ADR reporting, pharmacovigilance and methods about ADR reporting.

After educational intervention all nurses (100\%) were aware about the method of ADRs reporting. No nurse was aware about TTM before study. However, 20\% nurses had used this method for detection of ADR, but they didn't know the name of method. Only $13.3 \%$ nurses opined that all healthcare professional can report ADRs but after test all nurses $(100 \%)$ were aware that all healthcare professional, including them can report ADRs.

About the question of the which kind of ADRs should be reported, $11(36.6 \%)$ nurses opined reporting of ADRs due to blood products, $26.6 \%$ opined-ADRs to old drug, $13.3 \%$ opined nurses don't report ADRs, $23.3 \%$ opined that only serious life threatening ADRs in pre-test. In post-test all opined that all kind of ADRs should be reported with old drugs, life threatening related to blood product.

Vomiting, rash, diarrhea, oedema- these are the common ADRs observed by them. Sudden stoppage of the drug and new drug administration were the most common triggers observed by them

\section{DISCUSSION}

Nurse can play an important role in detecting and reporting of adverse drug reaction as being in close contact with patients. Spontaneous method is commonly used method for reporting of ADRs. However, spontaneous method has disadvantages like under reporting and difficulty in detection of delayed ADRs. Trigger tools have certain advantages over the spontaneous method like early detection and incidence can be obtained. Nurses are aware about only commonly used spontaneous method of ADRs reporting. Educational interventions about trigger tool method eventually increase the awareness about the need for safety of drug treatment. The IHI global trigger tool is a universal trigger tool adopted by researchers across the world. ${ }^{7}$ Trigger tools are known to vary as per the availability of drugs and prescribing pattern in a patient population. A study conducted by Abideen $\mathrm{P}$ et al, in India recommended a modified trigger tool in context to a hospital setup in India. ${ }^{6}$ In the present study, authors prepared an indigenous trigger tool list that has been adopted from the IHI global trigger tool and the tool used by Abideen $\mathrm{P}$ et al, and given to participant nurses. The proposed trigger tool comprised of drug trigger, laboratory trigger and patient triggers.

A total 30 nurses from medicine indoor wards were included for this study. Total 17 potential triggers were given to nurses, of which 14 triggers were detected by them in the study population. Of these, $11(64.7 \%)$ triggers were associated with an ADR and helped to detect these ADRs. A study conducted in Torrento by Matlow A et al, suggested that 34 triggers out of 94 triggers were useful in detecting ADRs. ${ }^{15}$ A study conducted in Hyderabad in medicine department by Yeramilli A et al, evaluated 100 ADRs and found that seven out of 17 selected triggers were related to an ADR. ${ }^{16}$ The above variation in results suggests that trigger tool needs to be customized and modified in each health care setting.

Most commonly observed triggers in this study were antiemetics, antacids, new drug administration, antidiarrheals. A study conducted by Rozich et al, showed that most commonly observed triggers were droperidol, abrupt medication stop, diphenhydramine, rash. ${ }^{17}$ In this study, the overall PPV of the trigger was $20.76 \%$. The PPV of individual triggers is a pointer to its value as a trigger tool. The individual PPV of each trigger ranged in this study from 0 to $100 \%$. In another study, it was observed that PPV for individual trigger ranges from $15 \%$ to $92.5 \% .{ }^{18} \mathrm{PPV}$ ranges from $0 \%$ to $88.3 \%$ for individual triggers was found in a study conducted by Matlow et al. ${ }^{9}$ While the overall PPV of trigger tool observed in studies conducted by Haffner et al, Rozenfeld $\mathrm{S}$ et al, Takata et al, were $18.6 \%, 14.4 \%$ and $16.8 \%$ respectively. ${ }^{8,19,20}$ 
The overall positive predictive value in this study therefore compares well with similar studies by other researchers. Patient triggers showed higher PPV as compared to drug trigger and laboratory trigger in this study. Triggers with higher PPV may lead to better detection and reporting of ADR. Occurrence of higher frequency of TT with a higher PPV is associated with higher rate of detection of ADRs. But, the PPV is highly influenced by the prevalence of AEs. Low PPV may be due to poor trigger performance or low event rates.

Of the 22 ADRs detected by nurses using TT method, gastrointestinal system was the most common affected system followed by injection site disorder, skin. Blood products, NSAIDs drugs were the common causal drugs followed by anti-tubercular drugs, antibiotics and antiretroviral. Results observed by Rozenfield $\mathrm{S}$ et al, showed that medicines most commonly implicated were tramadol, glibenclamide and furosemide. Moreover, the most commonly affected systems were CVS (27.8\%), CNS $(22.2 \%)$ and GIT $(20.4 \%) .{ }^{8}$ A study conducted by Iris L and Allison $\mathrm{K}$ et al, observed that the most common drugs implicated in ADRs were antibiotics. ${ }^{21}$

An Indian study revealed that drugs commonly implicated in ADR were cephalosporins followed by anti-diabetic agents. ${ }^{16}$ The variation from the reference list is attributed to possible differences in disease pattern and drug prescribing pattern. However, the absence of certain triggers does not imply that these triggers are invalid.

In this study, 11 out of 17 triggers showed the potential to detect ADRs. The predictive value and usefulness of the remaining triggers needs to be evaluated in future studies. As most of the ADRs were probable, which indicate the understanding and reporting of nurses about TTM and ADRs.

Selection of only a single department from the hospital with small population and limited number of triggers are limitations of this study. The utility of the triggers with low PPV can be further evaluated by studies in a larger patient population and in other departments of the hospital.

\section{CONCLUSION}

TTM is an effective method of ADR reporting in the healthcare settings. Its efficacy is good when it was used by nurses. The TTM helps to detect and report ADRs by nurses. Educational interventions about TTM will be helpful in better detection and reporting of ADRs. Further research is required to explore the feasibility and validity of TTM in different health care settings.

\section{Funding: No funding sources} Conflict of interest: None declared

Ethical approval: The study was approved by the Institutional Ethics Committee

\section{REFERENCES}

1. Satoskar RS, Bhadarkar SD, Rege NN. General pharmacology. In: Satoskar RR, eds. Pharmacology and Pharmacotherapeutics. 22nd ed. 2011;3:40.

2. Murphy BM, Frigo LC. Development, implementation, a results of a successful multidisciplinary adverse drug reaction reporting program in a university teaching hospital. Hosp Pharm. 1993;28:1199-204.

3. Ramesh M, Pandit J, Parthasarathi G. Adverse drug reactions in a south Indian hospital-their severity and cost involved. Pharmacoepidemiol Drug Safety. 2003;12(8):687-92.

4. Ganachari MS, Wadawa T, Walli S. Trigger tool for monitoring and reporting of adverse drug reactions a scientific tool for efficient reporting. Open Access Sci Rep. 2013;2(4):1-5.

5. Naessens JM, O’btrne TJ, Johnson MG. Measuring hospital adverse events: assessing inter-rater reliability and trigger performance of the Global Trigger Tool, Int J Quality Heal Care. 2010;22(4):266-74.

6. Abideen P. Practical implications of spontaneous adverse drug reaction reporting system in hospitals-an overview. Asian J Pharmaceut Clin Res. 2013;6(4):105 .

7. Naessens JM, O'byrne TJ, Johnson MG, Vansuch MB, McGlone CM, Huddleston JM. Measuring hospital adverse events: assessing inter-rater reliability and trigger performance of the Global Trigger Tool. Int $\mathbf{J}$ Quality Heal Care. 2010;22(4):266-74.

8. Rozenfeld S, Giordani F, Coelho S. Adverse drug events in hospital: pilot study with trigger tool. Pub Heal Magazine. 2013;47(6):1102-11.

9. Matlow A, Cronin C, Flintoft V, Nijssen-jorden C, Fleming M, Brady-Fryer B, et al. Description of the development and validation of the Canadian paediatric trigger tool. BMJ Qual Safety. 2011;20(5):416-23.

10. World Health Organization (WHO). The Importance on Pharmacovigilance. Safety Monitoring on Medicinal Products, Geneva (Switzerland): Office of Publications, World Health Organization. 2002.

11. Naranjo CA, Busto U, Sellers EM, Santor P, Ruiz I, Roberts EA, et al. A method for estimating the probability of adverse drug reactions. Clin Pharmacol Ther. 1981;30(2):239-45.

12. Hartwig SC, Siegel J, Schneider PJ. Preventability and severity assessment in reporting adverse drug reactions. Am J Heal Sys Pharm. 1992;49(9):2229-32.

13. Schumock G, Thornton JP. Focusing on the preventability of adverse drug reactions. Hosp Pharm. 1992;27:538.

14. Visser A, Slaman AE, Leijen CM, Gouma DJ, Goslings JC, Ubbink DT. Trigger tool versus verbal inventory to detect surgical complications. Langenbecks Arch Surg. 2015;400(7):821-30.

15. Matlow A, Cronin C, Flintoft V, Nijssen-jorden C, Fleming M, Brady-Fryer B, et al. Description of the development and validation of the Canadian paediatric trigger tool. BMJ Qual Safety. 2011;20(5):416-23. 
16. Yerramilli A, Veerla S, Chintala E, Guduguntal M, Vellivelli P, Sharma S, et al. A pharmacovigilance study using Tracer techniques. Adv Pharmacoepidemiol Drug Safety. 2014;3(4):1-5.

17. Lemon V, Stockwell DC. Automated detection of adverse events in children. Pediatric Clin North Am. 2012;59(6):1269-78.

18. Haffner S, Von LN, Wirth S, Thrmann P. Detecting adverse drug reactions on Peadiatric Wards. Drug Safety. 2005;28(5):453-64.

19. Takata G, Manson W, Taketomo C, Logsdon T, Sharek P. Development, testing and findings of a pediatric-focused trigger tool to identify medicationrelated harm in US children's hospitals. Pediatrics. 2008;121(4):e927-35.

20. Rozich JD, Haraden CR, Resar RK. Adverse drug event trigger tool: a practical methodology for measuring medication related harm. Quality Safety Heal Care. 2003;12(3):194-200.

21. Iris L, Alison k. Measuring adverse drug events on hospital medicine units with the institute for healthcare improvement trigger tool: a chart review. Can J Hosp Pharm. 2014;67(6):423-8.

Cite this article as: Meant UJ, Desai CK, Shah MK, Shah AN. Evaluation of trigger tool method for adverse drug reaction reporting by nursing staff at a tertiary care teaching hospital. Int J Basic Clin Pharmacol 2019;8:1139-44. 